\title{
Efficacy and safety of tacrolimus versus corticosteroid as initial monotherapy in adult-onset minimal change disease: a meta-analysis
}

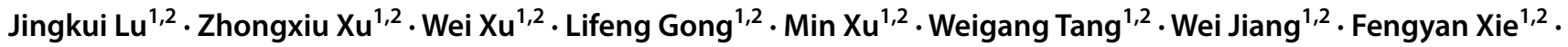 \\ Liping Ding ${ }^{1,2} \cdot$ Xiaoli Qian $^{1,2}$
}

Received: 7 May 2021 / Accepted: 11 January 2022 / Published online: 31 January 2022

(C) The Author(s) 2022

\begin{abstract}
Objective The objective of this meta-analysis was to compare the efficacy and safety of tacrolimus (TAC) monotherapy versus corticosteroid as initial monotherapy in adult-onset minimal change disease (MCD) patients.

Methods Databases including PubMed, Embase, the Cochrane Library, China National Knowledge Infrastructure, and Wanfang database were searched from the inception to March 20, 2021. Eligible studies comparing TAC monotherapy and corticosteroid as initial monotherapy for adult-onset MCD patients were included. Data were analyzed using Review Manager Version 5.3.

Results Four randomized controlled trials (RCTs) involving 196 patients were included in the meta-analysis. For initial monotherapy for adult-onset MCD, TAC and corticosteroid had similar complete remission (OR 1.06, 95\% CI 0.47-2.41, $P=0.89$ ), total remission (OR $1.30,95 \%$ CI $0.39-4.35, P=0.67$ ), relapse rate (OR $0.63,95 \%$ CI $0.28-1.42, P=0.26)$. Main drug-related adverse effects of two therapeutic regimens had no difference concerning infection (OR 0.54, 95\% CI 0.23-1.27, $P=0.15$ ), glucose intolerance (OR $0.55,95 \% \mathrm{CI} 0.16-1.84, P=0.33$ ) and acute renal failure (OR 1.37, 95\% CI 0.36-7.31, $P=0.71$ ).
\end{abstract}

Conclusion TAC monotherapy is comparable with corticosteroid monotherapy in initial therapy of MCD. To further confirm the conclusion, more large multicenter RCTs are necessary.

Keywords Tacrolimus $\cdot$ Corticosteroid $\cdot$ Minimal change disease $\cdot$ Meta-analysis

\section{Introduction}

Minimal change disease (MCD) causes up to $10-15 \%$ of primary nephrotic syndrome (NS) in adults [1]. Persistent NS results in infections, thromboembolic events, hyperlipidemia, cardiovascular disease [2-4]. Acute kidney injury accompanies the presentation of MCD in up to 20-25\% of cases [5]. High doses of corticosteroid have been

Jingkui Lu and Zhongxiu Xu have contributed equally to this work and should be considered as co-first authors.

Wei Xu

q844055361@qq.com

1 Department of Nephrology, Wujin Hospital Affiliated With Jiangsu University, Changzhou City 213000, Jiangsu Province, China

2 Department of Nephrology, The Wujin Clinical College of Xuzhou Medical University, Changzhou City 213000, Jiangsu Province, China recommended as initial therapy of MCD according to Kidney Disease Improving Global Outcomes (KDIGO). Compared with children, adults often have a delayed response to corticosteroid [6]. The relapse of adult-onset MCD is over $50 \%$. One-third of patients become frequent relapse or steroid-dependent [7]. This necessitates repeated or long-term use of corticosteroid. However, the adverse effects of highdose corticosteroid also become common, which include in cosmetic changes, weight gain, impaired glucose tolerance, osteoporosis, infection, and gastrointestinal bleeding [8, 9].

Tacrolimus (TAC), an immunosuppressive macrolide of calcineurin inhibitors (CIN) group, is a relatively attractive alternative to corticosteroid for treatment of MCD. TAC can suppresses IL-2 transcription, inhibit the growth and differentiation of $\mathrm{T}$ cells, thereby reduce the immune damage of podocyte finally [10]. Compared with another calcineurin inhibitors such as ciclosporin, TAC showed stronger immunosuppressive effect and fewer side effects [11-13]. Some studies reported successful TAC treatment of frequently 
relapsing, steroid-dependent, and steroid-resistant MCD [14, 15]. However, compared with corticosteroid, the efficacy of TAC monotherapy as the first-line initial agent in adult-onset MCD is uncertain. Our meta-analysis was conducted to compare the efficacy and safety between TAC with corticosteroid in initial monotherapy of adult-onset MCD.

\section{Materials and methods}

\section{Search strategy}

We searched PubMed, Embase, the Cochrane Library, China National Knowledge Infrastructure, and Wanfang database from the inception to March 20, 2021. The combined text and $\mathrm{MeSH}$ terms included minimal change disease, corticosteroid, and tacrolimus. In addition, the cited papers and relevant references were searched manually to identify eligible studies. There was no language restrictions.

\section{Inclusion criteria}

The inclusion criteria were defined as follows:

1. Randomized controlled trials (RCTs), cohort or casecontrol studies;

2. Age $>18$ years, NS, a kidney biopsy showing MCD and serum creatinine level of $<133 \mu \mathrm{mol} / \mathrm{L}$;

3. Studies were designed to compare TAC with corticosteroid as initial monotherapy for adult-onset MCD;

4. The main endpoint of the review was complete remission (CR) and total remission (TR). Secondary endpoints were relapse and drug-related adverse effects. $\mathrm{CR}$ is defined as proteinuria $<0.3 \mathrm{~g} /$ day with normal serum albumin and creatinine. Partial remission (PR) is defined as proteinuria $0.3-3.5 \mathrm{~g} /$ day which had declined to $\leq 50 \%$ of the baseline value. TR is defined as either $\mathrm{CR}$ or PR. Relapse is defined as proteinuria $>3.5 \mathrm{~g} / \mathrm{d}$ in patients who had achieved CR or PR.

\section{Exclusion criteria}

The exclusion criteria were defined as follows:

1. Case series, comments, reviews;

2. Lack of relevant outcomes data;

3. secondary minimal change disease, malignant tumour, infection (hepatitis B or $\mathrm{C}$ virus infection, tuberculosis and syphilis), diabetes mellitus, pregnancy, lactating, active gastrointestinal bleeding, other untreated infections, or any condition that would cause the study to be detrimental to the patient;

\section{Data extraction and quality assessment}

Data were extracted independently by two investigators using standard data extraction forms. In the case of disagreement, a third investigator was consulted. We extracted characteristics including first author, year of publication, location, study design, follow-up period, age, sex, sample size, specific drug treatment program, and outcomes. The Cochrane assessment tool was used to evaluate the quality of RCTs [16].

\section{Statistical analysis}

We performed the data analysis using Review Manager Version 5.3 (Cochrane Collaboration). Heterogeneity between studies was assessed using $I^{2}$ statistics. We considered $I^{2}>50 \%$ and $P<0.10$ to imply significant heterogeneity. Homogeneous data were performed using the fixed-effects model. Heterogeneous data were performed using the random-effects model. We presented categorical variables as Odds Ratios (OR). Summary estimates and $95 \%$ confidence intervals (CIs) were calculated. Overall effects were determined by the using $Z$ test. A $p$ value $<0.05$ was considered significant. Publication bias was assessed using sensitivity analysis.

\section{Results}

\section{Study selection and characteristics}

A flow diagram of the selection process is shown in Fig. 1. Finally, four RCTs were included in this analysis [17-20]. As a whole, 100 patients were included in TAC monotherapy group and 96 patients were included in steroid group. The follow-up period was from 36 to 82 weeks. The risks of bias in included RCTs were moderate. The baseline characteristics of these studies are listed in Table 1. Specific drug treatment program are listed in Table 2. The Cochrane assessment is listed in Table 3.

\section{Meta-analysis results}

\section{CR and TR}

Data about CR were reported in eight articles, 78/92 (84.8\%) for TAC group and 75/89 (84.2\%) for steroid group. The heterogeneity among studies was not substantial $\left(P=0.23, I^{2}=30 \%\right)$, so finally the fixed-effects model was used for the meta-analysis. There was no significant 
Fig. 1 Flow diagram of the literature search

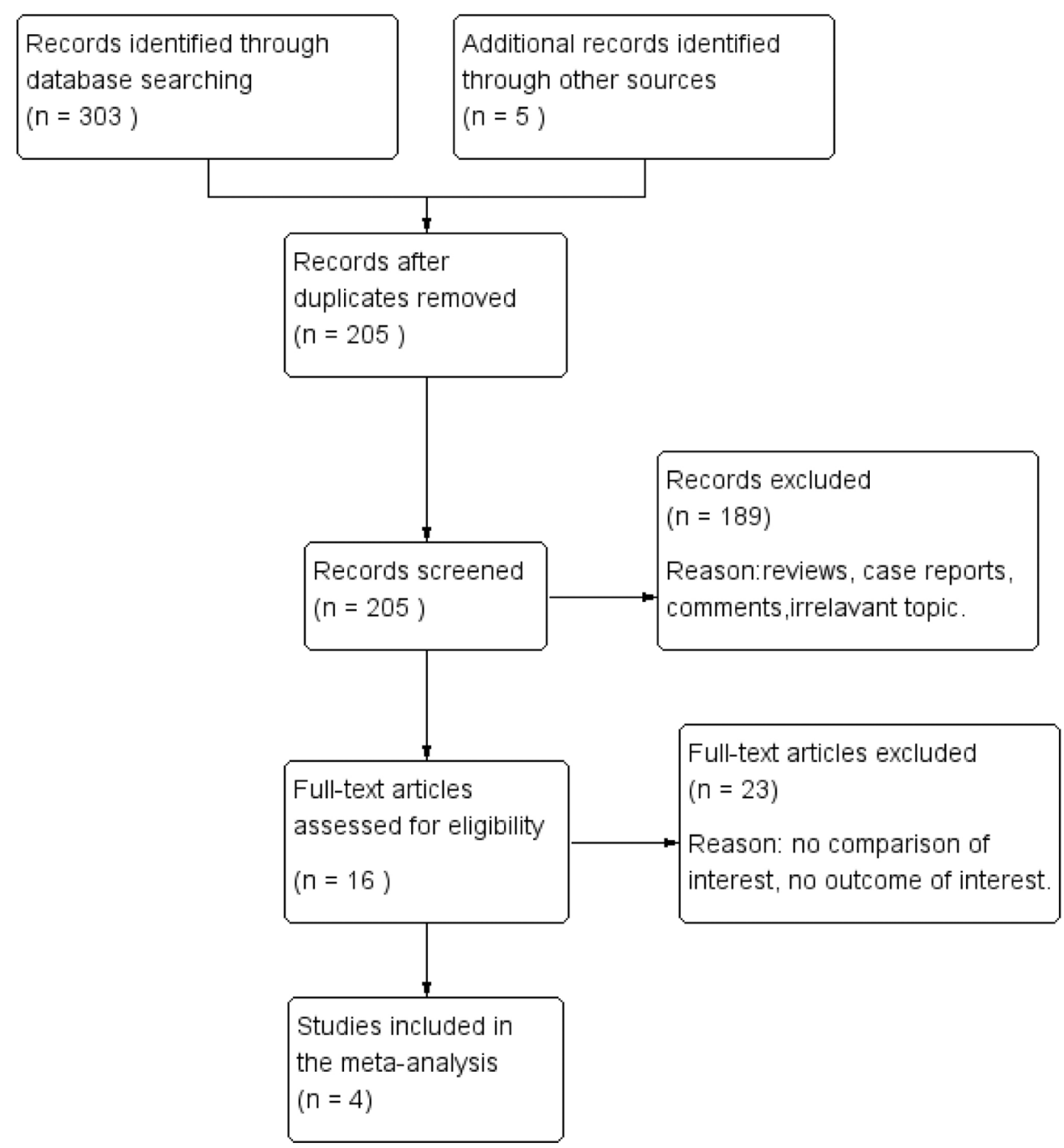

difference between two groups concerning CR (OR 1.06, 95\% CI 0.47-2.41, $P=0.89$ ) (Fig. 2).

Data about TR were reported in three articles, 60/66 (90.9\%) for TAC group and 58/65 (89.2\%) for steroid group. The heterogeneity among studies was not substantial $\left(P=0.66, I^{2}=0 \%\right)$, so finally the fixed-effects model was used for the meta-analysis. There was no significant difference between two groups concerning TR (OR 1.30, 95\% CI 0.39-4.35, $P=0.67$ ) (Fig. 3).

\section{Relapse rate}

Data about relapse rate were reported in three articles, 26/68 (38.2\%) for TAC group and 30/65 (46.2\%) for steroid group. The heterogeneity among studies was not substantial $\left(P=0.91, I^{2}=0 \%\right)$, so finally the fixed-effects model was used for the meta-analysis. Relapse rate of steroid group was higher than TAC group, but the difference was not statistically significant (OR $0.63,95 \%$ CI $0.28-1.42, P=0.26$ ) (Fig. 4).

\section{Drug-related adverse effects}

Data about main drug-related adverse effects were reported in four articles. Incidences of infection $(10.6 \%, 10 / 94)$, glucose intolerance $(4.3 \%, 4 / 94)$, acute renal failure $(4.5 \%$, $3 / 67)$, were in TAC group. Incidences of infection $(18.0 \%$, $16 / 89)$, glucose intolerance $(7.9 \%, 7 / 89)$, acute renal failure $(3.1 \%, 2 / 64)$, were in steroid group. There was no statistical significant difference between the two groups concerning infection (OR $0.54,95 \%$ CI $0.23-1.27, P=0.15$ ), glucose intolerance (OR 0.55, 95\% CI 0.16-1.84, $P=0.33$ ) and acute renal failure (OR $1.37,95 \% \mathrm{CI} 0.36-7.31, P=0.71$ ). All forest plots of drug-related adverse effects are listed in Figs. 5, 6,7 .

\section{Sensitivity analyses}

The sensitivity analyses for all outcomes after the two therapy regimens were used to judge the dependability of the 


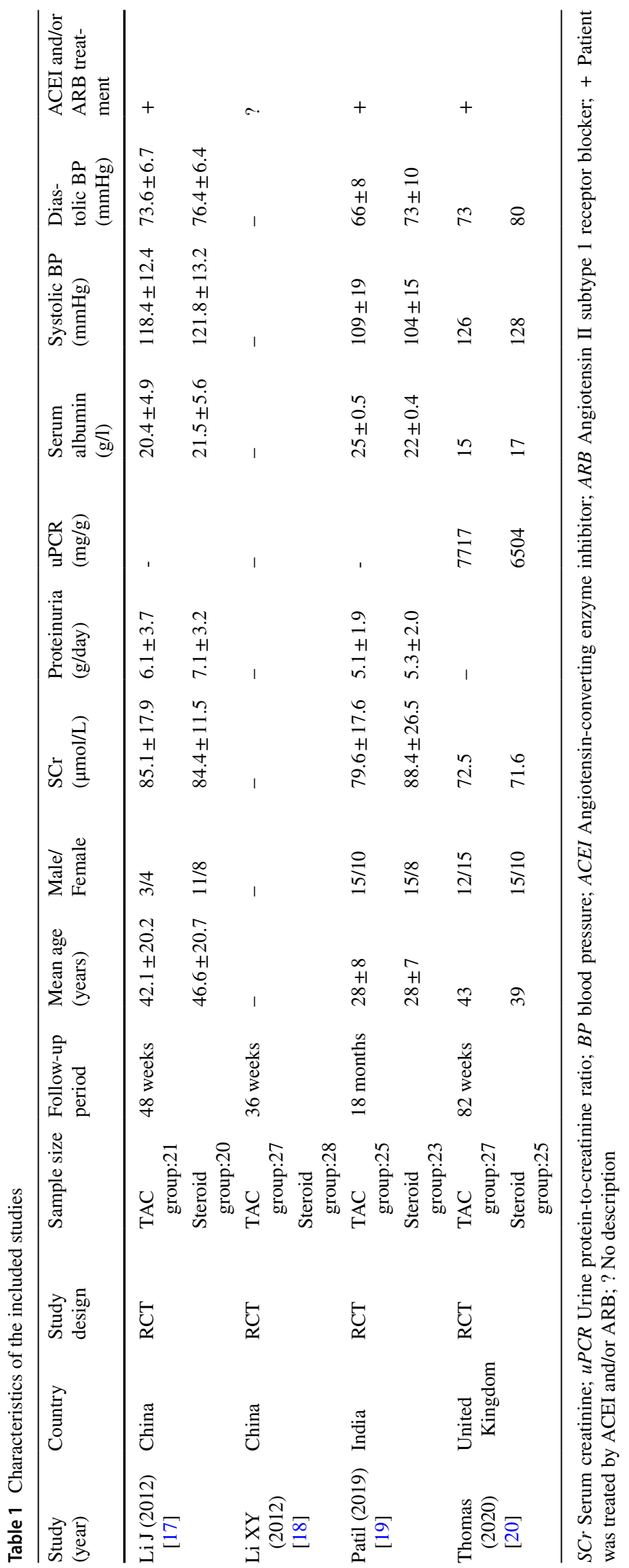




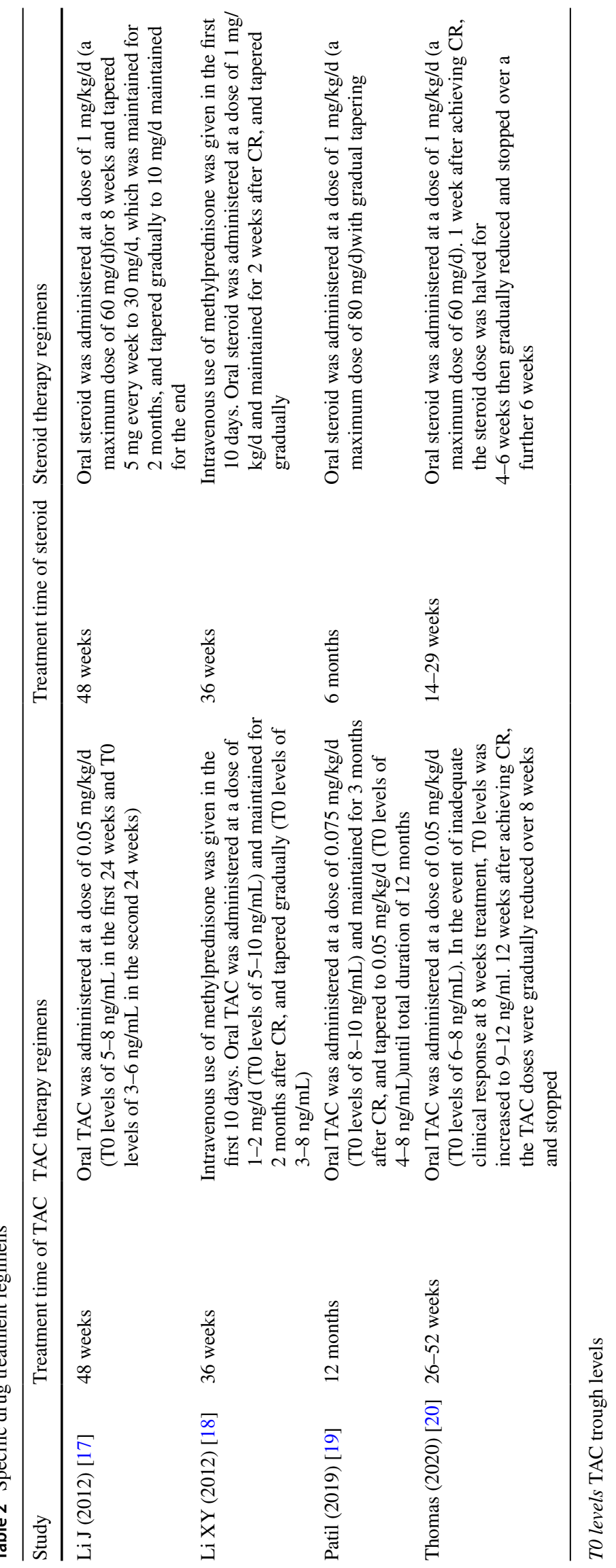


Table 3 Quality assessment of RCTs

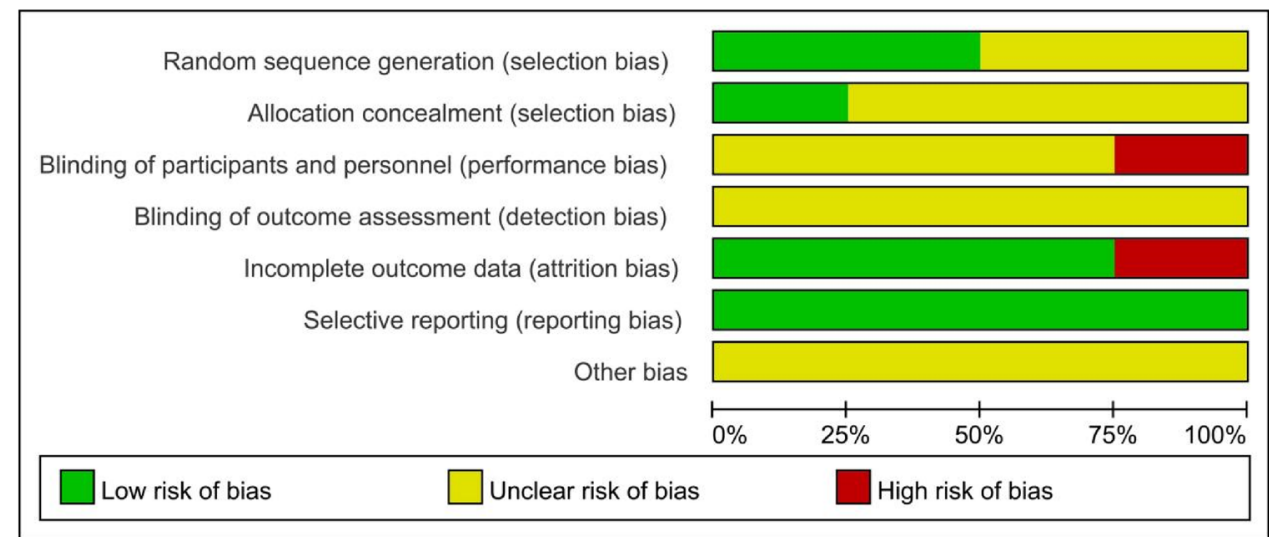

TAC

Steroid

Odds Ratio

Study or Subgroup Events Total Events Total Weight $\mathrm{M}-\mathrm{H}$. Fixed, $95 \% \mathrm{Cl}$

Li J 2012

Li XY 2012

Patil 2019

Thomas 2020

$20 \quad 21$

$20 \quad 21$

$16 \quad 25$

$\begin{array}{lll}17 & 20 & 7.4 \%\end{array}$

$17 \quad 21 \quad 7.3 \%$

$18 \quad 23 \quad 60.5 \%$

$22 \quad 25$

Total $(95 \% \mathrm{CI})$

Total events

92

Heterogeneity: $\mathrm{Chi}^{2}=4.28, \mathrm{df}=3(\mathrm{P}=0.23) ; \mathrm{I}^{2}=30 \%$

Test for overall effect: $Z=0.14(P=0.89)$

$3.53[0.34,37.15]$

$4.71[0.48,46.22]$

$0.49[0.14,1.78]$

$0.64[0.10,4.19]$

$89100.0 \%$

$1.06[0.47,2.41]$ 75

Fig. 2 Forest plots comparing CR between TAC and steroid group

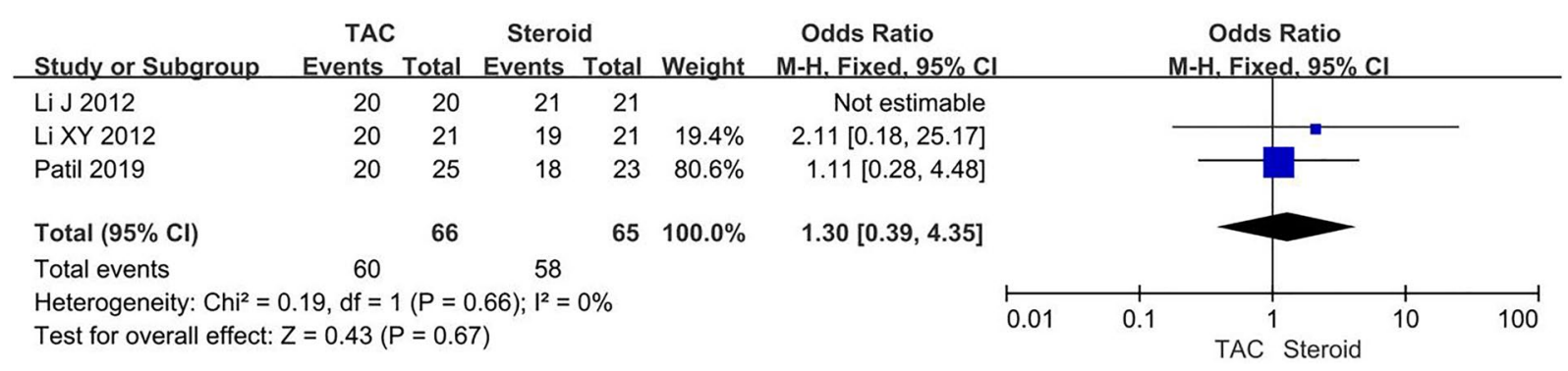

Fig. 3 Forest plots comparing TR between TAC and steroid group

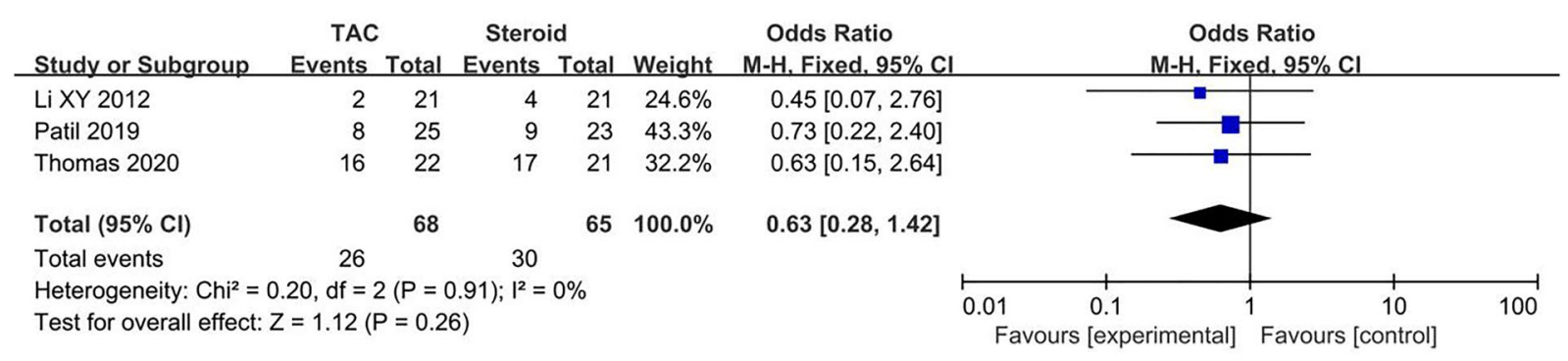

Fig. 4 Forest plots comparing relapse rate between TAC and steroid group 


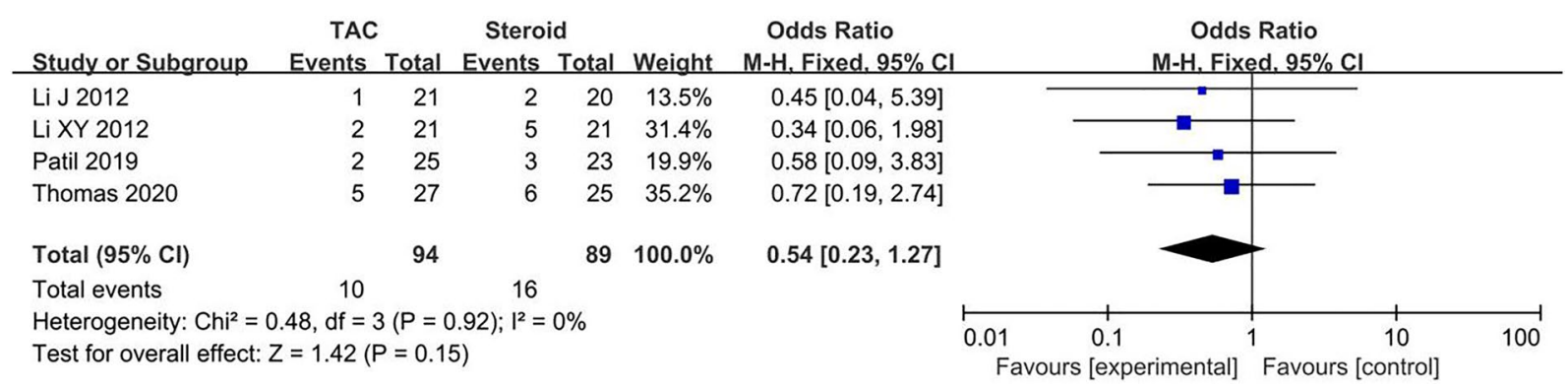

Fig. 5 Forest plots comparing infection between TAC and steroid group

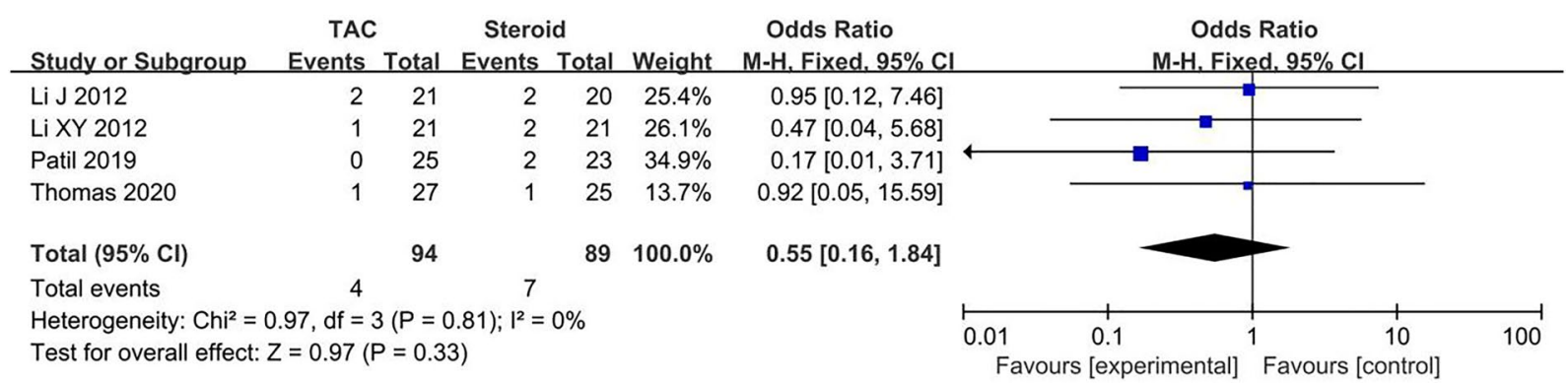

Fig. 6 Forest plots comparing glucose intolerance between TAC and steroid group

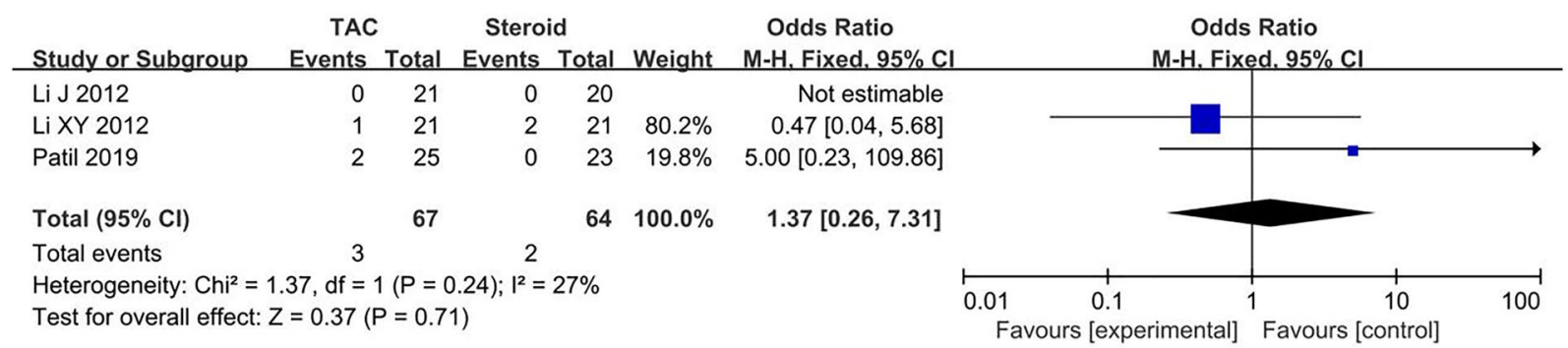

Fig. 7 Forest plots comparing acute renal failure between TAC and steroid group

results. We deleted one study at a time, the results of metaanalysis still showed no difference.

\section{Discussion}

Corticosteroids are recommended as initial therapy of MCD, which is effective [6]. At the same time, the adverse effects of corticosteroids are significant, so the clinicians need consider alternative treatments. The current guidelines lack advice on the effectiveness of steroid-free regimens used as initial therapy of MCD. In some studies, TAC was reported to be effective in treatment of frequently relapsing, steroiddependent, and steroid-resistant MCD [21-23]. Our metaanalysis was conducted to compare the efficacy and safety between TAC monotherapy with corticosteroid monotherapy for initial therapy of MCD. We found that TAC monotherapy is comparable with corticosteroid monotherapy for MCD concerning remission, relapse and drug-related adverse effects.

At present, corticosteroid monotherapy is effective in the treatment of MCD, which can achieve CR in $80 \%$ of patients with MCD [6]. Our meta-analysis showed that CR of corticosteroid monotherapy group was $84.2 \%$ and was comparable to the remission rates reported in other studies. In our meta-analysis TAC monotherapy also achieved high remission rates of $84.8 \%$. In Thomas study included in our meta-analysis, treatment times of TAC or corticosteroid were relatively short, but CR of the two treatment method were both high and close to $90 \%$. In general, TAC 
monotherapy was comparable to corticosteroid monotherapy concerning remission of NS.

MCD is easy to recur. Our meta-analysis showed that relapse rates were similar in corticosteroid and TAC treatment groups, which were lower than the $48-76 \%$ relapse rates reported in other studies [5, 9, 24, 25]. In Li Xiayu and Patil study included in our meta-analysis, the followup time was not long enough, which might not reflect the final relapse rates. Regimens of longer treatment time and higher dose may decrease relapse rates [20].

Long-term use of corticosteroid therapy can increase the incidence of drug-related adverse effects, so clinicians should evaluate the beneficial and adverse effects when prescribing treatment regimens for MCD patients. There are some significant unpleasant cosmetic drugrelated adverse effects associated with corticosteroid such as obesity, acne, striae, and moon facies, which can be debilitating for patients, especially the young adults, and have an impact on adherence with treatment. Neither of these cosmetic drug-related adverse effects occurred in the TAC treatment. Concerning other common adverse effects, such as infection, glucose intolerance, acute renal failure, TAC and corticosteroid have no significant difference. The nephrotoxicity of TAC is of great concern. It has been reported in the literature that the nephrotoxicity of TAC is related to its dose and concentration. The initial dose of TAC was $0.15 \mathrm{mg} / \mathrm{kg} / \mathrm{d}$, which can lead to acute reversible nephrotoxicity. The initial dose of TAC was $0.08 \mathrm{mg} / \mathrm{kg} / \mathrm{d}$, which do not occur nephrotoxicity [26].

There were some limitations in our meta-analysis. First, there were some differences concerning the specific treatment regimen and definition of outcomes, which might affect the heterogeneity among the studies. Second, most included studies had small sample size and the number of included studies was small, so our meta-analysis may not be adequate to judge effectiveness and safety of the two treatment.

\section{Conclusions}

Our meta-analysis revealed TAC monotherapy is comparable with corticosteroid monotherapy in initial therapy of MCD. To further confirm the conclusion, more large multicenter RCTs are necessary.

Funding The research was supported by the Wujin District Project Foundation (No.WS202011), the Young Talent Development Plan of Changzhou Health Commission (No. CZQM2021026) and the Science and Technology Development Foundation of the affiliate hospitals of Xuzhou Medical university (No. XYFY2021023).

\section{Declarations}

Conflict of interest The authors declare that they have no conflict of interest.

Open Access This article is licensed under a Creative Commons Attribution 4.0 International License, which permits use, sharing, adaptation, distribution and reproduction in any medium or format, as long as you give appropriate credit to the original author(s) and the source, provide a link to the Creative Commons licence, and indicate if changes were made. The images or other third party material in this article are included in the article's Creative Commons licence, unless indicated otherwise in a credit line to the material. If material is not included in the article's Creative Commons licence and your intended use is not permitted by statutory regulation or exceeds the permitted use, you will need to obtain permission directly from the copyright holder. To view a copy of this licence, visit http://creativecommons.org/licenses/by/4.0/.

\section{References}

1. Hull RP, Goldsmith DJ (2008) Nephrotic syndrome in adults. BMJ 336:1185-1189

2. Huang JJ, Hsu SC, Chen FF (2001) Adult-onset minimal change disease among Taiwanese: clinical features, therapeutic response, and prognosis. Am J Nephrol 21:28-34

3. Kerlin BA, Ayoob R, Smoyer WE (2012) Epidemiology and pathophysiology of nephrotic syndrome-associated thromboembolic disease. Clin J Am Soc Nephrol 7:513-520

4. Radhakrishnan J, Appel AS, Valeri A (1993) The nephrotic syndrome, lipids, and risk factors for cardiovascular disease. Am J Kidney Dis 22:135-142

5. Waldman M, Crew RJ, Valeri A (2007) Adult minimal-change disease: clinical characteristics, treatment, and outcomes. Clin J Am Soc Nephrol 2:445-453

6. KDIGO (2012) Clinical practice guidelines for glomerulonephritis. kidney disease: improving global outcomes. Kidney Int Suppl 2:181-185

7. Tse KC, Lam MF, Yip PS (2003) Idiopathic minimal change nephrotic syndrome in older adults: steroid responsiveness and pattern of relapses. Nephrol Dial Transplant 18(7):1316-1320

8. Shinzawa M, Yamamoto R, Nagasawa Y (2014) Comparison of methylprednisolone plus prednisolone with prednisolone alone as initial treatment in adult-onset minimal change disease: a retrospective cohort study. Clin J Am Soc Nephrol 9:1040-1048

9. Eguchi A, Takei T, Yoshida T (2010) Combined cyclosporine and prednisolone therapy in adult patients with the first relapse of minimal-change nephrotic syndrome. Nephrol Dial Transplant 25:124-129

10. Rauch MC, San Martín A (2009) Tacrolimus causes a blockage of protein secretion which reinforces its immunosuppressive activity and also explains some of its toxic side-effects. Transpl Immunol 22:72-81

11. Jardine AG (2005) Assessing the relative risk of cardiovascu lar disease among renal transplant patients receiving tacrolimus or cyclosporine. Transpl Int 18:379-384

12. Grimm M, Rinaldi M, Yonan NA (2006) Superior prevention of acute rejection by tacrolimus vs. cyclosporine in heart transplant recipients-a large European trial. Am J Transplant 6:1387-1397

13. Choudhry S, Bagga A, Hari P (2009) Effificacy and safety of tacrolimus versus cyclosporine in children with steroid-resistant nephrotic syndrome: a randomized controlled trial. Am J Kidney Dis 53:760-769 
14. Li H, Shi X, Shen H (2012) Tacrolimus versus intravenous pulse cyclophosphamide therapy in Chinese adults with steroid-resistant idiopathic minimal change nephropathy: a multicenter, open-label, nonrandomized cohort trial. Clin Ther 34:1112-1120

15. Li X, Li H, Chen J (2008) Tacrolimus as a steroid-sparing agent for adults with steroid dependent minimal change nephrotic syndrome. Nephrol Dial Transplant 23:1919-1925

16. Furlan AD, Malmivaara A, Chou R (2015) 2015 Updated method guideline for systematic reviews in the Cochrane back and neck group. Spine 40:1660-1673

17. Li J, Yao LL, Jin L (2012) Clinical study of tacrolimus monotherapy in the treatment of minimal change nephrotic syndrome. Strait Pharm J 24(9):153-156

18. Li XY, Xu N, Chen JH (2012) Comparison of short-term intravenous methylprednisolone after tacrolimus monotherapy and standard steroid regimen in the treatment of adult-onset minimal change disease: a prospective, open, randomized, controlled, multi-center clinical study. Zhejiang nephrology academic annual conference proceedings. pp 161-162

19. Patil MR, Divyaveer SS, Raychaudhary A (2019) Tacrolimus as the first-line agent in adult-onset minimal change disease: a randomized controlled study. Saudi J Kidney Dis Transpl 30(1):129-137

20. Medjeral-Thomas NR, Lawrence C, Condon M (2020) Randomized, controlled trial of tacrolimus and prednisolone monotherapy for adults with de novo minimal change disease: a multicenter, randomized, controlled trial. Clin J Am Soc Nephrol 15(2):209-218
21. Westhoff TH, Schmidt S, Zidek W (2006) Tacrolimus in steroidresis- $t$ ant and st eroid-dependent nephrotic syndrome. Clin Neph rol 65(6):393-400

22. Loeffler K, Gowrishankar M, Yiu V (2004) Tacrolimus therapy in pediatric patients with treatment-resist ant nephrotic syndrome. Pediatr Nephrol 19(3):281-287

23. Sinha MD, Macleod R, Rigby E (2006) Treatment of severe st eroid-de-pendent nephrotic syndrome (SDNS)in children with tacrolimus. Nephrol Dial T ransplant 21(7):1848-1854

24. Szeto CC, Lai FM, Chow KM (2015) Long-term outcome of biopsy-proven minimal change nephropathy in Chinese adults. Am J Kidney Dis 65:710-718

25. Szeto CC, Lai FM, To KF (2001) The natural history of immunoglobulin a nephropathy among patients with hematuria and minimal proteinuria. Am J Med 110:434-437

26. Segarra A, Vila J, Pou L (2002) Combined therapy of tacrolimus and corticosteroids in cyclosporin-resistant or -dependent idiopathic focal glomerulosclerosis: a preliminary uncontrolled study with prospective follow-up. Nephrol Dial Transplant 17(4):655-662

Publisher's Note Springer Nature remains neutral with regard to jurisdictional claims in published maps and institutional affiliations. 